\title{
OBSTRUCTIVE SLEEP APNEA: A REVIEW IN ADULTS
}

\author{
K. Elavarasi ${ }^{1}$
}

\section{HOW TO CITE THIS ARTICLE:}

K. Elavarasi. "Obstructive Sleep Apnea: A Review in Adults". Journal of Evolution of Medical and Dental Sciences 2014; Vol. 3, Issue 28, July 14; Page: 7881-7890, DOI: 10.14260/jemds/2014/2987

ABSTRACT: Obstructive sleep apnea (OSA) is the most prevalent sleep disorder in the adult population. There is accumulating evidence that OSA is being considered as an independent risk factor for hypertension, diabetes mellitus, cardiovascular diseases and stroke leading to increased cardio metabolic morbidity and mortality. The prevalence of OSA is higher in patients presenting for surgery than in general population and a significant proportion of OSA patients remain undiagnosed, when they present for surgery. This is of concern to the anesthesiologist and the peri-operative physician, as OSA has been associated with increased peri-operative risk and post-operative complications. Hence a protocol designed to provide practical solutions and strategies for the perioperative /post-operative care of these patients needs to be followed and optimal use of it requires our attention. Early recognition and treatment of OSA may prevent from adverse health consequences. A multidisciplinary approach to peri-operative care and constant vigilance by experienced anesthesia providers is paramount to ensuring positive patient outcome.

KEYWORDS: Obesity, sensitivity to anesthetic agents, peri-operative intra-operative and postoperative risks, obstructive sleep apnea.

INTRODUCTION: OSA is the most common apnea in obese individuals. It is characterized by repetitive, partial or complete collapse of the upper airway during sleep, causing impaired gaseous exchange and sleep disturbances. It also causes serious pathological changes in body systems. OSA is present in approximately $11.4 \%$ of men and $4.7 \%$ of women worldwide. ${ }^{(1,2)}$

OSA is defined according to the apnea / hypoapnea index (AHI) or respiratory disturbance index (RDI). As per the American Academy of Sleep medicine recommendations OSA is defined with $\mathrm{AHI}>5$, and it is classified as mild OSA of 5 to 15 , moderate OSA with 16 to 30; and severe OSA with $\mathrm{AHI}>30 .{ }^{(3)}$

Symptoms associated with OSA include snoring, excessive day time somnolence and restless sleep. Aging, obesity, male gender, smoking, alcohol consumption, family history, genetic predisposition, trauma, craniofacial/congenital anomalies like Pierre robin's syndrome are known factors associated with OSA. $(4,5,6)$

According to Fietze and colleagues(7) ${ }^{(7)}$ over the course of next 5 years the incidence of moderate OSA may increase by $8 \%$.Chung and colleagues ${ }^{(8)}$ have noted the prevalence of OSA is higher in patients presenting for surgery than in general population and significant proportion of these patients remain undiagnosed, when they present for surgery. Gupta and colleagues (9) have found that OSA patients undergoing hip or knee replacement were at an increased risk of developing peri-operative complications (24\% versus $9 \%$ respectively).

2006 guidelines published by American society of anesthesiologist (10) recommend the collaboration of anesthesiologist and primary physicians to develop an evaluation protocol prior to surgery. Proposed guidelines from the Adult obstructive sleep apnea task force of American academy of sleep medicine(3) ${ }^{(3)}$ ggests, pre-operative screening for OSA is a critical step in identifying patients 
at risk. However clinical practices pertaining to peri-operative management of OSA surgical patients are inconsistent and recent increased attention in this area has revealed a lack of uniformity surrounding practice guideline in the assessment and management of patients with OSA.

The relevant articles along with their references were searched and studied to provide a brief over view of pathophysiology of OSA with an emphasis on the potential peri-operative, intra and post- operative adverse events facing OSA patients and measures the anesthesiologists can take to remain vigilant and proactive in support of positive patient outcome.

ANATOMY OF OSA: According to Davidson (11) evolutionary changes to facilitate speech and language drove anatomical changes in upper respiratory tract, which led to the development of OSA.OSA occurs due to repetitive occlusion of the pharyngeal airway during sleep. OSA patients have structurally a more collapsible pharyngeal airway than non OSA persons. Understanding the mechanisms of the pharyngeal structural abnormalities is of great importance to develop new OSA treatments and for anesthesiologists to improve safety of peri-operative airway management.

OSA patients have excessive soft tissue surrounding the pharyngeal airway for a given maxilla- mandible size.(12) The pharynx connects to the trachea, which is pulled caudally during large tidal inspiration. Pharyngeal airway patency is dependent on breathing.(13) An increase in lung volume, decreases the collapsibility of the pharyngeal airway and apnea - hypo apnea index in OSA patients.

Reduced lung volume in obese OSA patients possibly leads to decrease in longitudinal tension of the pharyngeal airway increasing collapsibility of the pharynx. ${ }^{(14,13)}$ Anesthetic agents used during surgery decreases pharyngeal tone and depress the ventilatory responses to hypoxia and hypercapnia; thus it is essential that anesthesiologists identify the potential anatomical mechanisms compensating for the anatomical abnormalities after surgery.

\section{SYMPTOMS AND DIAGNOSTIC CRITERIA FOR OSA:}

SYMPTOMS: 1) Snoring 2) daytime sleepiness or fatigue 3) head ache on awakening in the morning 4) poor concentration 5) loss of memory 6) depression or mental irritability 7) Dry mouth or sore throat upon awakening 8) night perspirations 9) restless during sleep10) sexual dysfunction 11) Sudden awakening from sleep with a sensation of gasping or choking.

PHYSICAL EXAMINATION: Examination by itself cannot allow an accurate diagnosis of OSAH 'S, but it does help to exclude other causes for the patient's symptoms. Weight and height should be documented on the first visit and continue to document weight at all subsequent visits.

Approximately $50 \%$ of patients with OSAH'S are obese (BMI> $30 \mathrm{~kg} / \mathrm{m} 2$ ). Neck circumference of patients with OSAH'S is often $>17$ " (43cms). We should visually inspect for abnormally small mandible, assess nasal patency, visually assess upper airway for obvious obstruction using indirect laryngoscopy.

Inspect for macroglossia, assess dentition and the presence or absence of teeth. Also assess pharyngeal appearance (tonsillar size, uvular appearance, and lumen size, measure blood pressure with an appropriate size cuff.

Perform routine respiratory, cardiovascular, and neurological examination to detect any coexisting disease like (e.g. cor-pulmonale, chest wall deformity, myopathies) as clinically indicated. 


\section{REVIEW ARTICLE}

Measure forced expiratory volume (FEV1) and forced vital capacity (FVC) to detect any significant spirometric abnormalities. Possibility of hypothyroidism, acromegally and marfan's syndrome as underlying causes for OSAH'S should always be considered and thyroid function tests are often indicated.

DIAGNOSTIC CRITERIA FOR OSA ADULTS: Respiratory effort related arousal (RERA) is an event characterized by increasing respiratory effort for 10 seconds or longer leading to an arousal from sleep, but one that does not full fill the criteria for a hypoapnea or apnea the criterion standard to measure RERAS is esophageal manometry.

1) Sleep test or poly somnogram (PSG) may be used for conformation. It is performed by trained technologist. This test will measure various body functions; it includes airflow, blood $\mathrm{O}_{2}$ levels, breathing patterns electrical activity of brain, eye movements, heart rate, and muscle activity.

\section{Table Diagnostic criteria for obstructive sleep apnea, adults}

$A, B$ and $D$ or $C$ and D satisty the criteria

A. At least one of the following applies:

1. The patient complains of unintentional sleep episodes during wakefulness, daytime sleepiness, unrefreshing sleep, fatigue, or insomnia.

2. The patient wakes with breath holding, gasping, or choking.

3. The bed partner reports loud snoring, breathing interruptions or both during the patient's sleep.

B. Polysomnographic recording shows the following:

1. Five or more scoreable respiratory events (i.e., apnea, hypopneas, or RERAs) per hour of sleep.

2. Evidence of respiratory effort during all or a portion of each respiratory event (In the case of a RERA, this is best seen with the use of esophageal manometry.)

or

C. Polysomnographic recording shows the following:

1. Fifteen or more scoreable respiratory events (i.e., apneas, hypopneas, or RERAs) per hour of sleep.

2. Evidence of respiratory effort during all or a portion of each respiratory event.

(In the case of a RERA, this is best seen with the use of esophageal manometry.)

D. The disorder is not better explained by another sleep disorder, medical or neurological disorder, medication use, or substance use disorder.

(American Academy of Sleep Medicine (AASM): ICSD-2, 2005.)

\section{Severity criteria}

Mild: 5 or 15 events per hour.

Moderate: 15 to 30 events per hour.

Severe: greater than 30 events per hour.

1) Nocturnal sleep pulseoximetry: The oxygen de saturation index (ODI) measures the mean number of episode of oxygen de saturation per estimated sleep hour and sometimes used as a surrogate for the AHI.

2) Epsworth sleepiness scale (ESS): used to measure excessive day time sleepiness, maximum score is 24.(15) The score can be used to clinically subdivide the patients into either normal range (ESS < 11-14), mild subjective day time sleepiness (ESS 15-18)or severe subjective daytime sleepiness (ESS > 18). ESS can also be used to predict the likelihood of long term compliance with nasal CPAP. 


\section{SYSTEMIC EFFECTS:}

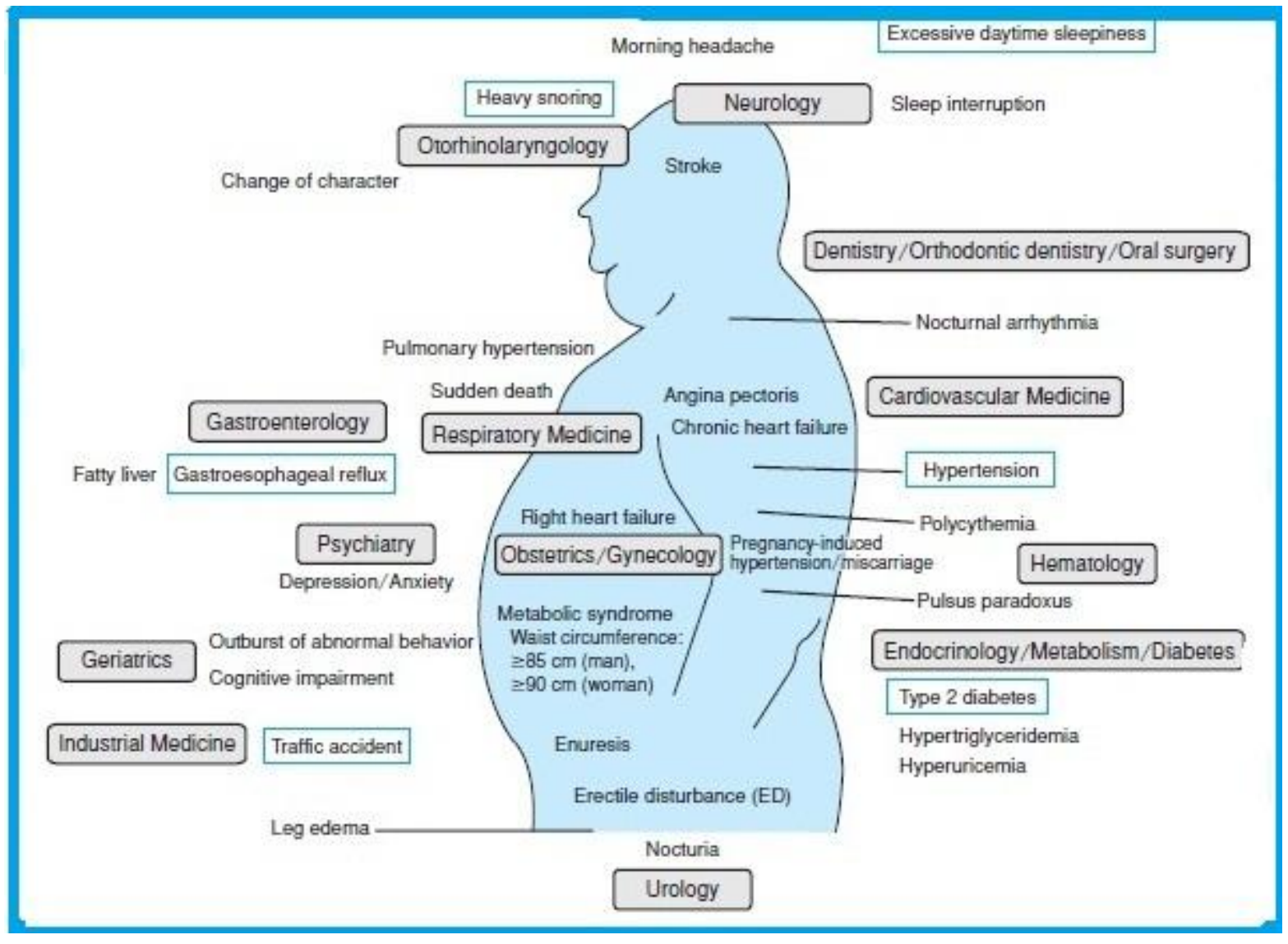

FIG. 1: SYSTEMIC EFFECTS IN OSA(16)

1) Neuro-behavioural and social: Excessive daytime sleepiness, impaired vigilance, mood disturbance, and cognitive dysfunctions are features of OSAH'S. The sleepiness interferes with efficiency of work and may worsen inter personal and social relationships. Partners of patients with OSA'S will be the first one who takes the patient to a doctor for evaluation of his/her sleep disorder.(16)

2) Cardiovascular: The intermittent hypoxia, chronic hypercarbia, frequent negative intra thoracic pressure variations, and sleep disturbance and arousals lead to increase in blood pressure and lead on to sustained hypertension via chronically heightened sympathetic nervous system activity and arterial baroreceptor dysfunction. Myocardial infarctions are also common.(16)

3) Central nervous system: is also affected, making patients prone for CVA'S and intracranial hypertension. Cardiac arrhythmias and corpulmonale are common in them.(16)

4) Diabetes mellitus: OSA is an independent risk factor for development for diabetes, associated with insulin resistance, obesity further increase the risk.(16)

5) Liver: Hepatic dysfunction marked by raised liver enzymes, fatty liver and fibrosis can be detected.(16) 
PRE OPERATIVE RISKS AND MANAGEMENT: Utilizing a short questionnaire with a simple portable device may be cost effective way to discover patients with high probability of OSA. Combining this pre-operative screening information with observations in the post anesthesia care unit may provide enough information to assist in proper post-operative patient care.(17)

Proper screening measures and pre-operative preparation can include the use of home sleep tests as a diagnostic tool for OSA. While the diagnostic gold standard remains the polysomnagraphy (PSG), it is expensive and time consuming. Pulse oximetry as a single metric of sleep apnea lacks the sensitivity and specificity of PSG and multi-channel home sleep testing. But pulse-oximetry can be considered along with apnea, hypoapnea index of 15 or 20 or more.

One best review was done in a national coverage determination in 2009 by centers for medicare and Medicaid services, with the final decision supporting equally effective testing utilizing PSG and home sleep tests, as measured by outcomes and patient compliance.(18)

Patients with mild OSA may not require pre-operative PAP therapy, but patients with moderate to severe OSA who have been on PAP therapy should continue treatment in the peri operative period. According to Laio and colleagues,(19) patients who have been non complaint with instruction for CPAP use prior to surgery and are in need of CPAP post-surgery, will pose the highest risk of complications.

However, Kaw and colleagues (20) found that CPAP use prior to surgery did not affect postoperative outcome. Due to patients diversity there is a need for attentive intra operative solutions to improve patient's outcome.

INTRAOPERATIVE RISK AND MANAGEMENT: Intra operatively OSA patients can have several issues to be dealt with including difficult intubation, opioid related respiratory depression and excessive sedation. Sedative pre medication should be avoided alpha-2 adrenergic agonist (Clonidine, Dexmeditomidine) may reduce intra operative anesthetic requirements and have opioid sparing effect. The two important correlates of difficult intubation are a higher mallampati score $\geq 3$, neck circumference $>40 \mathrm{cms}$, or waist circumference $>105 \mathrm{cms}$ (42") same for both genders.

Proper positioning should include supporting the obese patient behind the upper back and head to achieve an anatomical position, where a horizontal plane between the sternal notch and the external auditory meatus is established. Ramping/sitting posture decreases the frequency of airway obstruction compared to supine position.

This is required for adequate pre oxygenation and anesthetic induction, as obese patients have reduced FRC and tidal volume, often fall below the closing capacity of the small airways leading to atelectasis, increased intra pulmonary shunting and impaired oxygenation. FRC is further reduced after induction of anesthesia, when weight of the anterior chest compresses the thorax. At least 3 minutes of breathing $100 \% 02$ or 5 vital capacity breaths of $100 \% 02$ are essential.

ASA grading and difficult airway algorithm to be kept in mind and prepare appropriately for the management of difficult airway.

Consider proton pump inhibitors, antacids, rapid sequence induction with cricoid pressure. As these patients have opioid related respiratory depression; minimize use of opioids for analgesia, but short acting agents like remifentanil can be used. Regional and multi modal analgesia (NSAID'S, Acetaminophen, Dexmeditomidine, Dexamethasone) can be used. 
These patients can have carry over sedation effects from longer acting intravenous sedatives and inhaled anesthetic agents; hence use of Propofol /Remifentanil for maintainance of anesthesia is appropriate. Regional blocks, insoluble potent anesthetic agents (Desflurane, Sevoflurane) can be used. Use of intra operative capnography for monitoring of respiration is suggested.

Excessive sedation in monitored anesthetic care to be avoided. Anesthesia reversal can present problem with airway obstruction and desaturation. The upper airway collapses frequently during sleep and anesthesia. Upper airway patency depends on the balance between airway collapsing and dilating forces.

The current evidence suggests that patients who are prone to airway collapse have an anatomical predisposition to pharyngeal collapse on biomechanical basis, which is associated with an increased propensity of the supraglottic airway to collapse during inspiration.

In fact during contraction of the respiratory muscles (diaphragm and intercostals muscles), a negative airway pressure is being generated and a collapsible upper airway segment surrounded by soft tissues would collapse in the absence of its dilating forces. Post-operative residual curarization is common after administration of neuromuscular blocking agents.

There is a growing body of evidence that residual neuromuscular blockade places patients at increased risk.(21) Hence verification of full reversal of neuromuscular blockade is a must and ensure that the patient is fully conscious prior to extubation and placing the patient in semi -upright recovery position needs to be stressed.

The intra operative anesthesia team should be alerted in advance and peri operative OSA precautions should be taken. Future studies need to show which combination of anesthetics and opioid are optimal to achieve a persistently patent airway.

It is possible that drugs that increase respiratory drive such as $\mathrm{CO} 2$ or ampakins protect against fentanyl induced respiratory depression and lethal apnea in rats may be helpful to improve safety. Last but not the least to resume use of positive airway pressure device.

POST OPERATIVE RISKS AND MANAGEMENT: Patients who are at high risk of OSA on the screening questionnaires, and have recurrent post anesthesia care unit (PACU) respiratory events, are associated with higher post-operative respiratory complication. Diagnosed OSA patients previously on PAP therapy should be encouraged to be compliant with PAP therapy post operatively. Also, it may be prudent to monitor oximetry or telemetry monitoring.

In regard to length of hospital stay following surgery Gupta and colleagues (9) found that OSA patients remained in hospital significantly longer than control patients. OSA patients undergoing cardiac surgery had longer stay in ICU than control patients. Night can be particularly vulnerable time for patients with OSA, as sleep and sedation from critical respiratory depression appear very similar.

In order to ensure patient safety close monitoring, when narcotics are required may be necessary throughout the night. Moreover, by monitoring OSA patients for a longer time period post operatively, adverse outcomes such as post-operative airway problems, respiratory failure, ischemia, delirium, and death may be avoided.(22,23)

While current monitoring practices support the continuous monitoring in PACU and frequent monitoring on post op day 1. Patients with OSA may still have hypoxia for the first few post-operative days. Electronic monitoring is one possible solution though it has its own advantages and 
disadvantages. Ultimately the monitoring system used must be un-encumbering; transducers must be small and preferably wireless.

Home OSA screening manufacturers have made great advances in miniaturizing technology, which may be portable.(23) It is imperative that monitoring of at risk patients requiring narcotics continue for several days following surgery. By engaging in pre-operative screening, it is less likely that patients with undiagnosed OSA will suffer complications post operatively.

Regarding ambulatory surgery, short acting anesthetic agents and non-invasive surgery typically makes this a safer option for OSA patients. However, severe untreated or undiagnosed OSA requiring post-operative narcotics after ambulatory surgery may be unsafe.

OSA AND MALPRACTICE: Adverse outcomes can occur due to difficulties in intubation (due to increased oropharyngeal classification, decreased atlanto occipital extension, thick neck and occasionally micrognathia and retrognathia) and extubation difficulties (due to loss of optimal airway management, position, monitors off patients, patient not fully awake, no oropharyngeal airway) and together constitute approximately $30 \%$ of the malpractice OSA cases. However the prototypical OSA malpractice case constituting approximately $70 \%$ of the cases, is finding a post-operative patient dead in bed.

The clinical components of prototypical OSA malpractice " dead in bed " case are 1) Severe OSA 2) morbid obesity 3) patient in isolated ward / room 4) has no monitoring 5) is receiving narcotics 6) has a painful incision 7) is off oxygen 8) is off his or her PAP device.

Anesthesiologists, primary care doctors, sleep doctors and surgeons must have access to all OSA related information in OSA patients; it has been suggested that the best way to ensure this continuity of care is to issue medical alert bracelets to patients who have severe OSA. Even though initial cost may rise due to the increase in time and equipment needed to screen and diagnose patients ultimately, litigations will decrease due to improved patient care in the peri-operative environment.(24)

CONCLUSION: There may not be consensus regarding the best and cost effective methods for ensuring fewer peri-operative complications from OSA, there continues to be a need for informed clinicians, as patients are typically presenting with undiagnosed or misdiagnosed cases. Clinicians in peri-operative clinic can aid in identifying at risk patients by conducting screening tests in the clinical setting. At home sleep tests may also be useful in providing a preliminary identification of patients with OSA that need to be closely monitored in peri and post-operative setting.

Recent advances in the diagnostic tools of OSA, including simplified questionnaires and a myriad of inexpensive quick at home sleep tests have provided a necessary first step in assuring at an evidence based system for identifying OSA patients prior to the operating room settings, which can only serve to diminish the potentially negative outcomes associated with surgery and OSA. Postoperative management of OSA patients should include close monitoring postoperatively.

FUTURE DIRECTIONS: The problems of OSA, both in terms of prevalence and consequences are driving changes in the way OSA is investigated worldwide. Primary care physicians have become more adept at identifying the problem clinically, these resources have been overwhelmed and lengthy waiting lists have resulted. 
The expenses has also caused access problems for some. This has resulted in increased use of simple home based studies to triage the problem. These are suitable methods to rule in patients with a high pre- test probability on clinical grounds and may prove helpful to anesthesiologist seeking information quickly in patients presenting for pre anesthetic assessment.

Anesthesiologist also need to be aware that straight forward OSA is on the simple end of a spectrum of sleep related breathing disorders that include sleep hypoventilation and periodic breathing and can co-exist. But they require more detailed investigation.

OSA is widely prevalent problem and will become an increasing pre occupation of anesthesiologist, partly because as knowledge is developed in this area, the difficult airway is being seen in a broader context that just the peri-operative considerations. Prevalence is increasing with increasing obesity and aging.

Hence it gives an impetus to form a "Society of anesthesia and sleep medicine" to promote discussion, education, development of clinical standards and research related to these issues and to others common to anesthesia and sleep. This is a fertile area for exploration and enlightenment, and includes consideration of shared pathways determining unconsciousness, common effects of state change on ventilatory drive and reflex gain and the effects of anesthesia on and its interplay with post-operative sleep.

A multidisciplinary approach to peri-operative care is paramount to ensuring positive patient outcomes and there is much work to be done to improve understanding and clinical practice in these areas, both educational and research focused.

\section{REFERENCES:}

1. Bixler EO, Vgontzas AN, Ten HT et al. Effects of age on sleep apnea in men: 1 prevalence and severity. Amj respir crit care med 1998; 157: 144 - 8

2. Bixler EO, Vgontaz AN, Lin HM et al. Prevalence of sleep disordered breathing in women: Effect on gender. Amj respire crit care med 2001; 163: 608 -13

3. The Report of an American Academy of sleep medicine Task force. Sleep - related breathing disorders in adults :Recommendations for syndrome definition and measurement techniques in clinical research. Sleep 1999;21: 667 -89

4. Adult obstructive sleep apnea task force by the American Academy of sleep medicine clinical guideline for the evaluation, management and long term care of obstructive sleep apnea in adults. J clin Sleep med 2009; 5: 263 - 76.

5. Finkel KJ, Searleman AC, Tymkew H, et al. Prevalence of undiagnosed obstructive sleep apnea among adult surgical patients in an academic medical center. Sleep medicine. 2009; 10: 753 - 8.

6. Kaw R, Michota F, Jaffer A et al. Unrecognized sleep apnea in the obstructive sleep apnea patient chest 2006; 129: 198 - 205.

7. Fietze I, Penzel T, Alonderis A, et al. Management of obstructive sleep apnea in Europe. Sleep med.2011; 12 (2): 190 - 7.

8. Chung F, Yegneswaran B, Liaop, et al STOP questionnaire: a tool to screen patients for obstructive sleep apnea. Anesthesiology 2008; 108: 812 - 21. 


\section{REVIEW ARTICLE}

9. Gupta RM, Parvizi J, Hanssen AD, et al Post-operative complications in patients with obstructive sleep apnea syndrome undergoing hip or knee replacement: a case control study. Mayo clin proa. 2001; 76: 897 - 905.

10. 'Task force on peri-operative management of patients with obstructive sleep apnea: practice guidelines for peri-operative management of obstructive sleep apnea: a report by the American society of Anesthesiologists. Anesthesiology 2006; 104: 1081 - 93.

11. Davidson TM, The Great leap forward: the anatomic basis for the acquisition of speech and obstructive sleep apnea. Sleep med. 2003; 4: 185 _ 94.

12. Tsuiki S, Isonos, Ishikawa T, et al. Anatomical balance of the upper airway and obstructive sleep apnea. Anesthesiology. 2008; 108 (6): 1009 - 15.

13. Tagaito $Y$, Isonos S, Remmers J E, et al. Lung volume and collapsibility of the passive pharynx in patients with sleep - disordered - breathing. J Appl physiol. 2007: 103 (4):379 - 85.

14. Isonos. Obstructive sleep apnea of obese adults: Pathophysiology and peri operative airway management Anesthesiology. 2009; 110: 908 - 21.

15. ESS Johns M W. A new method for measuring day time sleepiness: The Epsworth leepiness scale. Sleep 1991; 14: 540 - 5.

16. G C Mbata and Chukwala ,Photo systemic effect -Obstructive sleep apnea hypoapnea syndrome Ann med health Sci Res. 2012 jan - jun; 2 (1): 74 - 77.

17. Chung F, Liao P. Pre-operative screening for obstructive sleep apnea - one death is too many Anaesth Intensive care. 2010; 38: 949 - 66.

18. Centers for medicare and Medicaid services Decision memo for sleep testing for obstructive sleep apnea (CAG - 00405 N). March 3, 2009 http: // www.cms.bhs.gov /mcd/ view decision memonasp? Id = 227. Accessed April 6, 2011.

19. Liao P, Yegneswaran B, Vairavanathan S et al, Post-operative complications in patients with obstructive sleep apnea: a retrospective matched cohort study. Can J Anesth. 2009; 56: 819 28.

20. Kaw R, Golish J, Ghamande S, et al Incremental risk of obstructive sleep apnea on cardiac surgical outcomes. J cardiovasc surg (torino) 2006; 47: 683-9.

21. Murphy GS, Szokol J W, Mary mont J H et al. Residual neuromuscular blockade and critical respiratory events in the post anesthesia care unit. Anesth Analg. 2008; 107 (1):130 - 7.

22. Kuniyoshi F H, Garlia - Touchard A, Gami AS, et al Day night variation of acute myocardial infarction in obstructive sleep apnea. J AM coll cardiol 2008; 52: 343 - 6.

23. Overdyk FJ, Carter R, Maddox RR, et al. Continuous oximetry, capnometry monitoring bradypnea during Patient controlled analgesia. Anesth Analg. 2007; 105 (2): 412 - 8.

24. Benumof JZ, Summary of prototypical OSA malpractice law case. Paper presented at: Challenges in the peri-operative management of OSA patients symposium; October 15, 2010; Sandiego, CA. 


\section{REVIEW ARTICLE}

\section{AUTHORS:}

1. K. Elavarasi

\section{PARTICULARS OF CONTRIBUTORS:}

1. Consultant, Department of Anaesthesiology, Vikram Hospital.

\section{NAME ADDRESS EMAIL ID OF THE}

\section{CORRESPONDING AUTHOR:}

Dr. K. Elavarasi,

No. $487,15^{\text {th }}$ Cross,

Indiranagar $2^{\text {nd }}$ Stage,

Bangalore-560038.

Email: chinnu123480@yahoo.com

Date of Submission: 26/06/2014.

Date of Peer Review: 27/06/2014.

Date of Acceptance: 07/07/2014.

Date of Publishing: 14/07/2014. 